\title{
Three years of experience with a novel "virtual" pneumonia follow-up clinic
}

\author{
To the Editor:
}

Community-acquired pneumonia (CAP) remains the leading cause of adult mortality from infectious disease in the developed world, and carries a huge economic burden [1]. The annual expenditure attributable to CAP is estimated at 10 billion euros and 10 billion dollars for hospitals in Europe and the USA, respectively [2]. Furthermore, older people are disproportionally affected by CAP, and the projected increases in the elderly population over the coming years will put increasing strain on health services [2].

Such pressures have resulted in the implementation of numerous cost-saving measures, such as reductions in the length of hospital stay, the use of less expensive antibiotics, and the stratification of patients to identify those who can be managed through ambulatory care [3, 4]. Patients admitted with severe pneumonia in the UK are typically followed-up in an outpatient clinic, 4-8 weeks after discharge from hospital, often with convalescent chest radiographs [5]. These are usually performed to ascertain whether the CAP was a complication of an underlying condition, such as cancer, and act as a baseline after recovery, in case the patient re-presents at a later stage $[6,7]$. A diagnosis of "severe" pneumonia is based on the judgement of the managing clinician as to the likelihood of adverse outcomes, and is usually based on a combination of clinical features and mortality risk scoring (such as CURB-65 or pneumonia severity index (PSI) scores) [8].

In 2013, a novel "virtual" pneumonia follow-up service was developed at our institution, in order to reduce outpatient workload and find a cost-effective, patient-friendly alternative to regular face-to-face clinics. The service formally invites patients to attend for a follow-up chest radiograph at 6 weeks (for patients aged $<80$ years) or 9 weeks (for those aged $\geqslant 80$ years) post-discharge. The reason for the longer follow-up duration for older patients is based on the observation that CAP is often more severe in older patients and may take longer to resolve $[9,10]$. The images are reviewed by a senior consultant respiratory physician (senior author) fortnightly, and the patients are either discharged, advised to return for further radiographs, or offered immediate outpatient follow-up. All radiographs also receive a formal report by a radiologist. Letters of correspondence are then sent to the patient and their primary care physician, to inform them of the outcome of the review.

We present the results from 3 years of experience with this service, using a database of severe pneumonia cases from 2013-2016 at University Hospital Southampton, UK. Using the electronic records of patients, we determined the number of patients who attended follow-up chest radiographs at 2 months, the proportion whose radiographs showed disease resolution and the number who needed further follow-up. Those that defaulted (did not attend) or required further imaging or follow-up were invited to attend at 3 or 4 months post-discharge.

The results can be viewed in table 1 . The virtual pneumonia follow-up service was well attended by patients, with 634 patients (83\%) receiving a convalescent chest radiograph within 2 months. Within 3 months, 675 (89\%) patients had been accounted for, and this increased to 692 (91\%) patients within 4 months. A total of 200 patients (26\%) either defaulted or had unresolved disease in the first round, and were followed-up in the second; whilst 100 (13\%) were followed-up in the third round.

Of 666 patients who had undergone imaging through the service within 4 months, 615 (92\%) showed resolved pneumonia on their radiographs and were able to be discharged without any further follow-up. Only 37 of these patients (6\%) required a further face-to-face outpatient clinical appointment for

@ERSpublications

A virtual pneumonia follow-up service provides significant efficiency savings and retains high quality patient care http://ow.ly/AUov30dRIXe

Cite this article as: Wilcox CR, Krishnan JV, Duffus C, et al. Three years of experience with a novel "virtual" pneumonia follow-up clinic. Eur Respir J 2017; 50: 1700703 [https://doi.org/10.1183/ 13993003.00703-2017]. 


\begin{tabular}{|c|c|c|c|c|c|}
\hline & Resolved (\%) & Unresolved (\%) & Defaulted (\%) & Other $(\%)$ & Clinic $(\%)$ \\
\hline 1st round ${ }^{\#}$ & $536(70)$ & $76(10)$ & $124(16)$ & $22(3)$ & $0(0)$ \\
\hline 2 nd round" & $60(8)$ & $13(2)$ & $87(11)$ & $4(0.5)$ & $34(4)$ \\
\hline 3 rd round ${ }^{+}$ & $19(2)$ & $0(0)$ & 70 (9) & $7(9)$ & $3(0.4)$ \\
\hline
\end{tabular}

Total patients $\mathrm{n}=762 .{ }^{*}$ : 2-month follow-up appointment; ${ }^{\text {I: }}$ 3-month follow-up appointment; ${ }^{+}$: 4-month follow-up appointment.

unresolved pneumonia. No abnormality was detected in five of these clinical patients. Three were found to have bronchiectasis, two were found to have interstitial lung disease, and two were found to have lung cancer. One patient each was found to have non-specific autoimmune disease, emphysema, asbestos-related pleural plaques, chronic lymphocytic leukaemia and bilateral hyatid cysts. One patient defaulted from clinical appointments, and one patient died before being seen.

A total of 33 patients (4\%) had other outcomes or conditions. 20 patients passed away before the completion of follow-up and eight patients were readmitted to hospital. Of these hospitalised patients, one was found to have lung cancer and two were found to have bronchiectasis. Further cross-sectional imaging and/or bronchoscopy was undertaken directly from the virtual clinic in five patients, without a prior face-to-face clinical referral. One patient each was found to have lung cancer, bronchiectasis, asbestos-related pleural plaques and emphysema.

Patient uptake and informal feedback has confirmed that this follow-up service is safe and time-efficient, and saves a significant number of outpatient clinic slots, making it both patient- and clinician-friendly. Formal patient feedback is currently being sought prospectively. Furthermore, the service is cost-effective, and we estimate that it saves approximately $60 \%$ of the outpatient tariff per patient (equating to $£ 100 / € 115$ per patient). This service has also helped in the early identification and management of numerous incidental pathologies (such as interstitial lung disease and malignancy), and acts as a valuable educational resource for trainees and students.

To conclude, given the enormous economic burden of caring for hospitalised patients with pneumonia, it is imperative that both treatment and follow-up services are streamlined to cope with increasing demand. We believe our novel service represents a viable strategy to provide efficiency savings, whilst retaining high quality patient care.

Christopher R. Wilcox @, Jamie V. Krishnan, Claire Duffus and Ben G. Marshall

Dept of Respiratory Medicine, University Hospital Southampton, Southampton, UK.

Correspondence: Christopher Wilcox, Dept of Respiratory Medicine, University Hospital Southampton, Tremona Road, Southampton, SO16 6DY, UK. E-mail: christopher.wilcox@soton.ac.uk

Received: April 032017 | Accepted after revision: June 282017

Support statement: No specific financial support was obtained for this study. Christopher Wilcox is supported by the National Institute of Health Research (NIHR) Academic Foundation Programme at the University of Southampton.

Conflict of interest: None declared.

\section{References}

1 Mandell LA, Wunderink RG, Anzueto A, et al. Infectious Diseases Society of America/American Thoracic Society consensus guidelines on the management of community-acquired pneumonia in adults. Clin Infect Dis 2007; 44: Suppl. 2, S27-S72.

2 Welte T, Torres A, Nathwani D. Clinical and economic burden of community-acquired pneumonia among adults in Europe. Thorax 2012; 67: 71-79.

3 Mertz D, Johnstone J. Modern management of community-acquired pneumonia: is it cost-effective and are outcomes acceptable? Curr Infect Dis Reports 2011; 13: 269-277.

4 Kosar F, Alici DE, Hacibedel B, et al. Burden of community-acquired pneumonia in adults over 18 years of age. Hum Vaccin Immunother 2017; 13: 1673-1680.

5 Lim WS, Baudouin SV, George RC, et al. BTS guidelines for the management of community acquired pneumonia in adults: update 2009. Thorax 2009; 64: Suppl. 3, iii1-ii55.

6 Dang TT, Eurich DT, Weir DL, et al. Rates and risk factors for recurrent pneumonia in patients hospitalized with community-acquired pneumonia: population-based prospective cohort study with 5 years of follow-up. Clin Infect Dis 2014; 59: 74-80. 
7 Holmberg H, Kragsbjerg P. Association of pneumonia and lung cancer: the value of convalescent chest radiography and follow-up. Scand J Infect Dis 1993; 25: 93-100.

8 Eccles S, Pincus C, Higgins B, et al. Diagnosis and management of community and hospital acquired pneumonia in adults: summary of NICE guidance. BMJ 2014; 349: g6722.

9 Kothe H, Bauer T, Marre R, et al. Outcome of community-acquired pneumonia: influence of age, residence status and antimicrobial treatment. Eur Respir J 2008; 32: 139-146.

10 Klapdor B, Ewig S, Pletz MW, et al. Community-acquired pneumonia in younger patients is an entity on its own. Eur Respir J 2012; 39: 1156-1161. 